\title{
Progressive Supranuclear Palsy: Postmortem Chemical Analysis
}

Over the past two decades, the postmortem analysis of brain neurotransmitters, neurohormones, metabolites, and neurotransmitter-related enzymes has yielded important information about the pathophysiological basis of human neurodegenerative disorders such as Parkinson's, Huntington's, and Alzheimer's diseases. Such studies have led to the investigation of new and more specific therapies for these degenerative diseases. Following in this issue, two independent groups of investigators report their findings on the postmortem neurochemistry of progressive supranuclear palsy (PSP). These studies by Kish's and Ruberg's groups shed light on the pathophysiological characteristics of PSP as well as on Parkinson's and Alzheimer's diseases.

PSP was first described clinically in 1904 [1] but was only defined as a specific clinicopathological entity in 1964 by Steele, Richardson, and Olszewski $[2,3]$. PSP is uncommon and accounts for about $4 \%$ of patients who manifest parkinsonian symptoms [1]. The onset of the disease is usually in the sixth decade, and it is somewhat more common in men than in women. The average duration of the disease is less than 10 years. This is similar to untreated Parkinson's disease, which has an average duration of about 7 to 10 years. The classic features of PSP are supranuclear ophthalmoparesis, bradykinesia, axial rigidity, postural instability, and pseudobulbar affect. Vertical-gaze paresis, especially down-gaze paresis, is the most common oculomotor sign. Bradykinesia and rigidity often develop early and can lead to an initial diagnosis of Parkinson's disease. In contrast to Parkinson's disease, however, tremor is unusual. Depression and irritability are common features, and dementia is frequently seen as the disease progresses.

The neurochemical abnormalities reported by Ruberg's and Kish's groups are in close agreement with each other. Their findings should enhance our understanding of basal ganglia function in general and of PSP in particular. Ruberg's group studied 9 cases of PSP, and Kish's group studied 5 cases. The most striking finding of both groups was a marked reduction in the concentrations of dopamine (80 to $90 \%$ ) and homovanillic acid (HVA) (45 to 50\%) in the caudate and putamen. Furthermore, the more marked reduction in dopamine concentrations as compared to HVA levels suggests an increased turnover of dopamine in the remaining dopamine terminals in the caudate and putamen. These observed reductions in dopamine and HVA concentrations are somewhat less marked than, but otherwise similar to, those seen in Parkinson's disease and provide a probable explanation for the axial rigidity and bradykinesia seen clinically in PSP.

In contrast to Parkinson's disease, however, both groups found normal dopamine and HVA levels in the nucleus accumbens. Kish and co-workers also measured norepinephrine and serotonin levels, which were generally normal. In Parkinson's disease, the loss of dopamine in the nucleus accumbens and limbic cortex has been hypothesized as a cause of depressive symptoms. This hypothesis is now in question because depression and dementia are common in PSP while dopaminergic function in mesolimbic and mesocortical pathways is normal. One would have to postulate either that there is a difference in the type or cause of depression seen in PSP versus Parkinson's disease or that the involvement of nonlimbic areas such as the caudate nucleus must be considered as an alternate explanation for the depression seen in these two diseases. In this regard, it is of interest that in Huntington's disease, depression is also very common, but, again, the nucleus accumbens is relatively spared.

Both groups of investigators measured choline acetyltransferase (CAT) activity in several brain regions. Ruberg and co-workers found a mild (approximately $20 \%$ ) decrease of CAT activity in the cerebral cortex; moderate decreases ( 40 to $60 \%$ ) in the caudate, putamen, and nucleus accumbens; and an even larger decrease $(70 \%)$ in the substantia innominata (the presumed source of a large number of cholinergic cortical afferents). In contrast, Kish and colleagues found no significant changes in CAT activity in the cerebral cortex or basal ganglia, even in 2 of the 5 PSP patients with documented severe dementia. This discrepancy must be resolved in further studies. Reported pathologic findings in the substantia innominata in PSP, although not as marked as those in Alzheimer's disease, are most consistent with the existence of at least some cholinergic deficits [4]. Neither group, however, found changes approaching the decreases in cerebral cortical CAT that have been found in $\mathrm{Alz}$ heimer's disease ( 85 to $95 \%$ ). Thus, it is unlikely that the dementia in PSP is caused primarily by cortical cholinergic deficits. Ruberg and colleagues suggest that the decreases in CAT activity in the substantia innominata are likely caused by a loss of cholinergic af- 
ferents to the substantia innominata from the brainstem. Such an explanation is consistent with the mild decrease in postsynaptic cholinergic receptors in the substantia innominata (approximately 26\%) and the equally mild changes in cortical CAT activity (approximately $21 \%$ ).

Ruberg and co-workers measured dopamine receptors using the potent D-2 antagonist $\left[{ }^{3} \mathrm{H}\right]$ spiperone. $\left[{ }^{3} \mathrm{H}\right]$ spiperone binding was decreased 40 to $50 \%$ in the caudate, putamen, and nucleus accumbens. Dopamine receptors were also decreased $34 \%$ in the frontal cortex and $67 \%$ in the substantia innominata. These decreases could be related either to drug therapy or to cell loss in the regions measured. Five of their PSP patients were treated until death with dopamine agonists, and all patients were treated with them at some point during their illness. Dopamine agonists may have caused a down-regulation in dopamine receptors throughout the brain. Alternatively, cell loss would lead to loss of receptors as most dopamine D-2 receptors are located on the intrinsic neurons that receive dopamine input. Cell loss would also provide an explanation for the loss of CAT activity in the caudate, putamen, and nucleus accumbens, structures that normally have large numbers of cholinergic interneurons. Ruberg and associates found a significant correlation between the decreases in CAT activity in various areas and the decreases in dopamine receptors. The loss of cholinergic interneurons in the basal ganglia may provide a possible explanation for the relative lack of effectiveness of dopamine agonists and cholinergic antagonists in PSP as compared with Parkinson's disease, as dopamine is thought to exert its pharmacological effects to a large extent through inhibition of cholinergic interneurons. The loss of cholinergic interneurons may also explain the lack of tremor in PSP. One of the most prominent effects of anticholinergic agents in Parkinson's disease is the relief of tremor. In Parkinson's disease, there is a relative underactivity of dopaminergic systems and an overactivity of cholinergic systems. In PSP, the loss of cholinergic markers in the basal ganglia tends to restore the dopaminergic/cholinergic balance. Because the cholinergic cells are postsynaptic to the dopamine cells, per- haps the efficacy of cholinergic agonists should be investigated in this disease.

Kish and co-workers found elevated glutamic acid levels in the temporal and occipital cortex, caudate, putamen, and medial globus pallidus. Levels of $\gamma$ aminobutyric acid (GABA) were found to be normal except in the subthalamic nucleus, where the GABA concentration was reduced. Glutamate is thought to be the neurotransmitter of corrical neurons projecting both to other areas of cerebral cortex and to various subcortical areas such as the caudate and putamen. Furthermore, glutamate has known neurotoxic effects in the brain. However, there is little evidence for a direct neurotoxic role of glutamate in PSP, as Kish and colleagues found no correlation between the degree of elevation of glutamate levels in a given structure and the severity of the neurological abnormalities in that region.

In summary, these two groups of investigators have presented valuable information that can help us to understand the parkinsonian symptoms, the lack of effectiveness of drug therapy, and the mild dementia observed clinically in PSP. Future studies should focus on the brainstem biochemical abnormalities, an understanding of which may help explain the ophthalmoparesis and pseudobulbar affect so common in this disorder.

Anne B. Young, $M D, P h D$

Department of Neurology

University of Michigan

Ann Arbor, MI 48104

\section{References}

1. Jankovic J: Progressive supranuclear palsy: clinical and pharmacologic update. Neurol Clin 2:473-486, 1984

2. Steele JC: Progressive supranuclear palsy. Brain 95:693-704, 1972

3. Steele JC, Richardson JC, Olszewski J: Progressive supranuclear palsy: a heterogenous degeneration involving the brainstem, basal ganglia and cerebellum with vertical gaze and pseudobulbar palsy, nuchal dystonia and dementia. Arch Neurol 10:333-359, 1964

4. Tagliavini F, Pilleri G, Bouras C, Constantinidis J: The basal nucleus of Meynert in patients with progressive supranuclear palsy. Neurosci Lett 44:37-42, 1984 